\title{
La comprensión del delirio a partir de la reformulación de los conceptos diferenciales de un sistema de creencias individual ${ }^{*}$
}

\section{The understanding of delusion through the reformulation of the distinguishing concepts of an individual belief system}

JOSÉ MARÍA ARISO

Universidad Internacional de la Rioja (España)

\section{RESUMEN}

Una de las líneas de investigación más interesantes de la actual filosofía de la psiquiatría gira en torno a la posibilidad de entender el contenido de los delirios a través de la noción wittgensteiniana de «certeza objetiva». En este artículo se mantiene que el contenido de los delirios se podría entender más fácilmente considerando la existencia de sistemas de creencias individuales y reformulando los conceptos diferenciales de dichos sistemas.

PALABRAS CLAVE

DELIRIO, CERTEZA OBJETIVA, CERTEZA SUBJETIVA, GRAMÁTICA, JUEGO DE

LENGUAJE

\footnotetext{
* Esta nota ha sido realizada dentro del marco del proyecto de investigación «Normatividad y Praxis: El debate actual después de Wittgenstein» (FFI2010-15975). 


\section{ABSTRACT}

One of the most interesting research lines in recent philosophy of psychiatry deals with the possibility of understanding the content of delusions through Wittgenstein's concept of «objective certainty». In this paper it is held that the content of delusions might be understood more easily by considering the existence of individual belief systems and reformulating the distinguishing concepts of such systems.

KEYWORDS
DELUSION, OBJECTIVE CERTAINTY, SUBJECTIVE CERTAINTY, GRAMMAR,
LANGUAGE-GAME

\section{INTRODUCCIÓN}

DesPuÉs DE QUE KaRL JASPERS PROClamaRa que «[e]s imposible comprender en su génesis un auténtico delirio», ${ }^{1}$ se aceptó de forma generalizada que las creaciones y producciones del sujeto delirante son totalmente inaccesibles a la genuina comprensión hermenéutica, lo cual provocó que desde comienzos del siglo XX los pacientes psicóticos fueran excluidos durante décadas del tratamiento psicoterapéutico. Ante esta tesitura, han surgido múltiples intentos de conceptualizar el delirio como un fenómeno comprensible, intentos que en los últimos años se vienen desarrollando sobre todo dentro del ámbito de la filosofía de la psiquiatría. Una de las líneas de investigación que están despertando más expectación en este campo es la que pretende comparar los delirios con la concepción de las certezas expuesta hace ya más de medio siglo por el filósofo vienés Ludwig Wittgenstein. Con el fin de aportar una alternativa dentro de esta línea de investigación, al comienzo de la presente nota resumiré tanto los logros como las limitaciones que han surgido al tratar de contemplar los delirios como certezas, tras lo cual analizaré la posibilidad de superar esas limitaciones, aunque sea parcialmente, entendiendo el delirio de dos maneras distintas: en primer lugar, como una certeza subjetiva, y en segundo lugar, como una certeza objetiva pero no colectiva sino meramente individual. De esta manera señalaré en qué sentido la noción de «certeza objetiva individual»-y la reformulación de los conceptos diferenciales implicados en este tipo de certezas- puede ser una herramienta conceptual útil para ayudarnos a comprender el contenido de los delirios.

1 K. Jaspers, Psicopatología general, tr. R. O. Saubidet y D. A. Santillán. México: FCE, 1999, p. 778. 


\section{ESTADO DE LA CUESTIÓN: \\ EL DELIRIO CONTEMPLADO DESDE LA OBRA DE WITTGENSTEIN}

El primer intento de contribuir a la comprensión del delirio partiendo de la filosofía tardía de Wittgenstein corrió a cargo de Fischer, el cual propuso que entender la esquizofrenia como una peculiar imagen del mundo a la cual correspondía una forma de vida distinta a la habitual permitiría reconocer la lógica interna de este trastorno, pues la realidad del esquizofrénico también estaría configurada por su lenguaje. Tal y como se acaba de señalar, la imagen del mundo del esquizofrénico no sería errónea sino simplemente distinta, ya que este sujeto conceptualizaría y categorizaría la realidad usando unas reglas distintas de las nuestras. ${ }^{2}$ Cualquier lector familiarizado con la obra tardía de Wittgenstein ya se habrá dado cuenta de que la terminología empleada por Fischer proviene en su mayor parte de las Investigaciones filosóficas; ${ }^{3}$ sin embargo, las alusiones más frecuentes e interesantes a la obra tardía de Wittgenstein en relación con los delirios no se dirigieron a las Investigaciones filosóficas sino a Sobre la certeza: ${ }^{4}$ y más concretamente, a las características de la noción misma de «certeza». Teniendo en cuenta que la American Psychiatric Association definió el delirio como una creencia falsa sostenida con firmeza a pesar de lo que casi todo el mundo cree y a pesar de todo aquello que constituya una prueba obvia e incontrovertible de lo contrario, ${ }^{5}$ Campbell señaló que las creencias delirantes tienen, para el sujeto que las padece, el mismo estatus epistemológico que las certezas wittgensteinianas. Así como las certezas -por ejemplo «Hay muchos objetos en el mundo», «El mundo existe desde hace mucho tiempo», etc.- no pueden ser empíricamente contrastadas porque conforman el trasfondo que permite confrontar una proposición con la realidad para dictaminar si aquella es verdadera o falsa, Campbell mantiene que los delirios de los pacientes con síndrome de Cotard -«Estoy muerto»-o Capgras -«No tengo cuerpo»- también constituyen la imagen del mundo en base a la cual estas personas conciben y perciben la realidad. ${ }^{6}$

2 H.R. Fischer, Sprache und Lebensform. Wittgenstein über Freud und die Geisteskrankheit. Heidelberg: Auer, 1991.

3 L. Wittgenstein, Investigaciones filosóficas, tr. A. García Suárez y U. Moulines. Barcelona: UNAM/Crítica, 1988. (En lo sucesivo, esta obra se citará con las siglas «IF»).

4 L. Wittgenstein, Sobre la certeza, tr. J. Ll. Prades y V. Raga. Barcelona: Gedisa, 1997. (En lo sucesivo esta obra se citará con las siglas «SC»).

5 American Psychiatric Association, Diagnostic Statistical Manual of Mental Disorders, $4^{\text {th }}$ edition, Text Revision (DSM-IV-TR). Washington DC: American Psychiatric Association, 2000, p. 765.

6 J. Campbell, «Rationality, meaning, and the analysis of delusion», Philosophy, Psychiatry, and Psychology, VIII (2001), pp. 89-100. 
Estos comentarios de Campbell no hicieron sino respaldar la opinión mantenida previamente por Eilan, autora que había advertido que la resistencia de los delirios a la evidencia contraria se debe precisamente al papel que comparten con las certezas como presupuestos básicos de los sistemas de creencias o certezas en los que se inscribe toda proposición. ${ }^{7}$ Desde el punto de vista de Eilan, nuestras certezas son las creencias fundamentales inmunes a la duda que configuran el trasfondo en el que tienen lugar nuestras inferencias y la interpretación misma de nuestras experiencias, en tanto que los delirios condicionan de igual manera nuestro razonamiento y la interpretación de la propia experiencia. ${ }^{8}$

Poco tiempo después, Thornton criticó la seguridad con que Campbell y Eilan habían equiparado los delirios con las certezas. Pues si bien Thornton reconoció que certezas y delirios comparten algunos rasgos epistémicos como los que he señalado anteriormente, advirtió que sólo podemos identificar nuestras certezas desde dentro de nuestro propio sistema, lo cual le llevó a concluir que la negación de cualquiera de nuestras certezas -negación que tendría lugar al considerar una certeza o sistema alternativo- sería inconcebible. ${ }^{9}$

\section{EL DELIRIO COMO CERTEZA SUBJETIVA}

Dejemos a un lado, aunque sea momentáneamente, los comentarios sobre la concepción de los delirios como certezas anormales descritos en el capítulo anterior y tratemos de retomar este asunto partiendo de cero. ¿Qué se podría decir sobre la concepción de los delirios como certezas? Para empezar, hay que tener en cuenta que Wittgenstein distingue entre certezas subjetivas y objetivas:

Con la palabra «cierto» expresamos la convicción absoluta, la ausencia de cualquier tipo de duda, y tratamos de convencer a los demás. Eso es certeza subjetiva.

Pero, ¿cuándo es una cosa objetivamente cierta? -Cuando el error no es posible. Pero, ¿qué tipo de posibilidad es éste? ¿No ha de quedar el error excluido lógicamente? ${ }^{10}$

Mientras que la certeza subjetiva carece de interés filosófico porque no va más de allá de la mera obstinación con que alguien trata de convencer a otra persona, la certeza objetiva tiene lugar cuando la posibilidad del error y de la

7 En lo sucesivo, y siguiendo a Wittgenstein ( $c f$. SC $\S \S 72,92,106-107,144,159$, etc.), utilizaré los términos «certeza» y «creencia» como sinónimos.

8 N. Eilan, «On understanding schizophrenia», en D. Zahavi (ed.), Exploring the self. Amsterdam: John Benjamins, 2000, pp. 97-113.

9 T. Thornton, «Why the idea of framework propositions cannot contribute to an understanding of delusions», Phenomenology and the Cognitive Sciences, VII (2008), pp. 159-175.

10 SC $§ 194$. 
misma duda está lógicamente excluida. En este caso, al igual que sucede en otros muchos pasajes de la obra tardía de Wittgenstein, el término «lógica» ya no se usa al modo clásico - tal y como se hacía en el propio Tractatus al hacer mención a la lógica tradicional aplicable a todos los mundos posibles- sino como un sinónimo de «gramática», ${ }_{11}^{1}$ expresión que Wittgenstein emplea para referirse a la totalidad de juegos de lenguaje compartidos por una comunidad lingüística. ${ }^{12}$ Así pues, una proposición será objetivamente cierta si en nuestros juegos de lenguaje ordinarios no hay lugar para posibilidad alguna de duda o error al respecto. A modo de ejemplo, un individuo podría repetir hasta la extenuación que está seguro de que el primer premio en el próximo sorteo de la lotería de Navidad recaerá en un número determinado alegando como única justificación que tiene una «corazonada»: naturalmente, en este caso la posibilidad de error permanece por mucho que el sujeto en cuestión se obstine e insista en convencernos de que su presentimiento se hará realidad. De hecho, su certeza subjetiva no se convertiría en objetiva aunque llegara a convencernos de que su «corazonada» se acabará cumpliendo ni aun cuando se cumpliera, pues la posibilidad de error al respecto persistirá sin variación alguna en nuestros juegos de lenguaje. En cambio, un sujeto tendrá certeza objetiva de que existen objetos físicos, de que la Tierra existe desde hace más de cinco minutos, etc., de modo que cualquier asomo de duda sobre estas cuestiones sería tomado como incomprensible.

A tenor de lo dicho, parece haber cuatro razones de peso por las que el delirio podría ser contemplado como una mera certeza subjetiva. En primer lugar, cabría pensar que el sujeto delirante pretende convencernos de la veracidad de su creencia delirante. En segundo lugar, y relacionado con lo anterior, el delirio podría tener un marcado componente de voluntariedad. En tercer lugar, parece obvio que el sujeto delirante estaría completamente equivocado. En cuarto lugar, y mucho más importante aún, si el delirio se contempla como una certeza parece evidente que ésta no puede ser objetiva, por lo que debería ser subjetiva. Analicemos cada una de estas razones. Respecto al primer argumento hay que decir que, al igual que nosotros no sentimos la necesidad de expresar nuestras certezas, el sujeto delirante tampoco necesita manifestar expresamente su delirio, entre otras cosas porque suele ser plenamente consciente de que podría provocar rechazo y exclusión social. En segundo lugar, es evidente que el delirio es ajeno a la voluntad del individuo, ya que la idea de un «delirio voluntario» resulta

$11 C f$. SC $\$ 628$.

12 Aunque Wittgenstein no ofreció una definición precisa de lo que entendía por «juego de lenguaje» ( $c f$.IF $\S 7$ ), podemos decir que con esta expresión se refirió a cada una de las prácticas en que se insertan nuestros usos del lenguaje, si bien en dichas prácticas hay que incluir también todas aquellas actividades con que aparecen entrelazados los usos del lenguaje. 
inconsistente en sí misma y sólo resulta inteligible si se diluye en un simple caso de fingimiento. En cuanto al tercer argumento, parece obvio que quien se muestra convencido de estar muerto o de no tener un cuerpo está simple y llanamente equivocado: ahora bien, semejantes «errores» son tan graves que ni siquiera pueden ser considerados como tales. El propio Wittgenstein advierte que los errores tienen un lugar concreto dentro de nuestros juegos de lenguaje, por lo que aquellos casos que no encajen dentro de esos lugares previamente establecidos no podrán ser considerados como errores sino como anomalías que se presentan ocasionalmente. ${ }^{13}$ Partiendo de aquí, salta a la vista que el delirio no es un mero error, sino una anomalía persistente que, al no tener cabida dentro de nuestros juegos de lenguaje ordinarios, no puede ser considerada como un error. Por último, las certezas son compartidas por una comunidad lingüística, en tanto que el delirio aparece como un caso individual que resulta a todas luces incompatible con las certezas comunitarias que el sujeto delirante compartía hasta que surgió el delirio. Dicho de otra manera, si el delirio fuera una certeza objetiva debería ser también colectivo o compartido por una comunidad, ya que la idea de una «certeza individual»-léase una «certeza objetiva individual»resulta totalmente ajena al contexto de la obra tardía de Wittgenstein. Al fin y al cabo, si se admitiera la existencia de certezas individuales y mutuamente incompatibles no habría margen para la posibilidad de un consenso que dotara de significado al lenguaje y no tendría sentido plantearse cuándo está lógica o gramaticalmente excluida la posibilidad del error, pues la gramática se constituye gracias al consenso prolongado de una comunidad de hablantes al tomar como absurda e incomprensible toda puesta en duda de una certeza. Por tanto, parece imposible manejar la idea de una «certeza individual» sin que ésta quede reducida a una certeza subjetiva; pero veamos hasta qué punto sería no sólo posible sino también útil referirse a una «certeza objetiva individual».

\section{EL DELIRIO COMO «CERTEZA OBJETIVA INDIVIDUAL»}

No cabe duda de que una de las principales características de la obra tardía de Wittgenstein es su carácter netamente descriptivo. Efectivamente, el pensador vienés entiende los problemas filosóficos como enredos conceptuales que se disolverán tras describir los equívocos usos del lenguaje que han generado la confusión. ${ }^{14}$ Ahora bien, si describimos con detalle determinados usos del lenguaje es posible que en alguna ocasión hallemos certezas allí donde no esperábamos encontrarlas. Así, es posible que una proposición del tipo «el azul es un color más frío que el verde» sea verdadera para una persona en un momento dado, falsa en otro, etc. Pero cabe la posibilidad de que alguien, 
un sujeto concreto, trate semejante proposición como una certeza, tratamiento que podría ser detectado atendiendo a la forma de hablar y actuar de este individuo. ${ }^{15}$ Aparentemente se trata de una certeza poco relevante porque su existencia no tendría una gran repercusión dentro del sistema de creencias, si bien su detección podría ayudarnos a conocer mejor la sensibilidad del sujeto en cuestión y, por extensión, su misma imagen del mundo. ${ }^{16}$ Mas también hay certezas individuales que pueden condicionar sobremanera la vida del sujeto en cuestión. Sirvan de ejemplo las certezas del tipo «Soy inferior a X». Algunos complejos de inferioridad pueden tornarse tan severos que la posibilidad de que exista duda o error alguno sobre este particular resulte no sólo inaceptable sino también totalmente incomprensible para el sujeto de turno: y no por pura testarudez u obcecación, sino porque esa creencia o certeza se ha apoderado de él. Asimismo, el uso del lenguaje que cabe apreciar en el sujeto afectado por el síndrome de Cotard revela su firme creencia de estar muerto, creencia que condicionará no sólo su uso del lenguaje sino también su imagen del mundo y su actuación en él.

Contemplar el delirio como una certeza puede ser sumamente útil en aquellos casos en los que la influencia del delirio parece extenderse de forma constante a todos los ámbitos de la vida del sujeto delirante, pues esta perspectiva puede ayudarnos a arrojar luz sobre la coherencia interna del delirio al ofrecer una panorámica global del sistema de creencias del sujeto. Pero tal y como apuntaron Bortolotti y Broome, los delirios están frecuentemente circunscritos a aspectos o momentos puntuales de la vida del individuo, en cuyo caso habrá contradicciones entre las certezas del mismo paciente: ${ }^{17}$ sin ir más lejos, Bayne y Pacherie señalaron que, en algunas versiones del síndrome de Capgras, los pacientes reconocen a menudo que el contenido de su delirio es sencillamente increíble. ${ }^{18}$ En este tipo de casos cabe plantearse la posibilidad de que la imagen del mundo del sujeto delirante no forme un todo coherente y unificado, por lo que apreciar las contradicciones existentes entre las distintas certezas de un sujeto

15 Recordemos una vez más que en este punto me alejo de Wittgenstein, pues según el pensador vienés (SC § 284), una proposición no se convierte en certeza objetiva por el carácter intrínseco de dicha proposición, sino porque es tratada como una certeza -o sea, porque su puesta en duda se considera absurda e incomprensible- por parte de una comunidad de hablantes.

16 Esta certeza puede resultar poco relevante para el hombre de la calle, pero repárese en la importancia que podría tener para alguien que, como Wassily Kandinsky, trató de revelar las relaciones internas entre los distintos colores. $C f$. W. Kandinsky, Cursos de la Bauhaus, tr. E. Sananes. Madrid: Alianza, 1991.

17 L. Bortolotti y M. Broome, «Delusional Beliefs and Reason Giving», Philosophical Psychology, XXI (2008), pp. 801-821.

18 T. Bayne y E. Pacherie, «Bottom up or top down», Philosophy, Psychiatry \& Psychology, XI (2004), pp. 1-11. 
podría ayudarnos a entender por qué a veces estos pacientes no muestran una pauta de acción constante o coherente con su delirio. La dificultad que surge en este punto es que, según dio a entender Wittgenstein, las certezas objetivas mutuamente incompatibles no pueden coexistir dentro de un mismo sistema: en los sistemas de creencias todas las certezas se sostienen recíprocamente, ${ }^{19}$ por lo que no hay margen para la contradicción entre ellas. Bortolotti y Broome intentaron solucionar este problema al apuntar que muchos delirios parecen estar circunscritos a una parte del sistema de creencias, como si se tratara de una diferencia muy puntual y localizada respecto al sistema de creencias característico de la comunidad lingüística a la que pertenece el sujeto delirante. ${ }^{20}$ Desgraciadamente, Bortolotti y Broome no aclaran cómo puede permanecer una certeza dentro de un sistema siendo incompatible con el resto de certezas de dicho sistema. Para entender lo que quiero decir, basta con preguntarse con qué certezas podría ser compatible la certeza de estar muerto, o bien retomar la objeción planteada por Thornton. Según vimos anteriormente, este autor advertía que el hecho de que no podamos abandonar la perspectiva o imagen del mundo correspondiente a nuestro sistema de creencias implica que no podemos identificar y menos aún entender certezas ajenas a las nuestras. Con el fin de aclarar esta observación de Thornton, me gustaría señalar que desconocemos totalmente cómo serían los juegos de lenguaje que podría jugar un muerto o lo que con ciertas resistencias podríamos denominar un «muerto viviente», por lo que las certezas de un muerto, en caso de que pudiera tenerlas, constituirían un completo enigma para nosotros. Hablando en rigor, no sabríamos qué esperar de un muerto. Ahora bien, tanto en los casos de síndrome de Cotard como en aquellas películas o ficciones en que se recrea la figura del «muerto viviente» apenas hay diferencia práctica alguna entre el «muerto viviente» y el ser vivo corriente, por lo que es evidente que cuando un paciente asegura que está «muerto» no usa dicho concepto del modo acostumbrado en nuestros juegos de lenguaje. Por tanto, el contenido de los delirios resulta incomprensible si lo analizamos partiendo de la premisa de que el significado de los términos empleados para expresar dicho contenido no difiere del significado que esos términos tienen habitualmente en nuestros juegos de lenguaje. El delirio sería total y absolutamente incomprensible si todas las palabras que expresen su contenido tuvieran un significado distinto del acostumbrado; pero en realidad, son sólo una selecta minoría de palabras o conceptos los que presentan dicho carácter diferencial. Además, cabe argumentar que hasta cierto punto sí entendemos al sujeto que dice no tener un cuerpo, estar muerto, etc. Pensamos que comete un craso error -aunque como señalé anteriormente, estas equivocaciones son demasiado graves como para

20 Bortolotti y Broome, loc. cit. 
ser consideradas meros errores-y observamos múltiples incoherencias entre su delirio y su comportamiento: por ejemplo, el hecho de que crea estar muerto y que hable, coma, camine, etc. Aunque no sepamos qué esperar de un muerto, entendemos al sujeto afectado por el síndrome de Cotard en la medida que le consideramos como un muerto «parcialmente» o sólo en algunos aspectos. Aquellos aspectos que no entendemos son precisamente aquellos que vienen determinados por los conceptos diferenciales del delirio: son conceptos con un uso similar al que de los mismos hacemos habitualmente, pero que al mismo tiempo pueden presentar notables diferencias y matices. Averiguar el uso que los sujetos delirantes hacen de estos conceptos diferenciales podría ayudarnos a entender el contenido de los delirios, cosa que a duras penas ocurre cuando el contenido del delirio se interpreta de forma literal. A modo de ejemplo, al analizar el lenguaje de un paciente que cree estar muerto tal vez pudiéramos vernos en la tesitura de tener que reformular el modo en que usa el concepto de «muerte», pues en determinados casos -o incluso por regla general-podría usar la expresión «Estoy muerto» de forma similar a como cualquiera de nosotros podría dar por hecho en una situación extrema que en unos breves instantes estará muerto, lo cual contribuiría a explicar también la notable ausencia de diferencias prácticas entre este sujeto delirante y cualquier otro individuo.

\section{A MODO DE CONCLUSIÓN}

Llegados a este punto, hay una pregunta fundamental cuya respuesta no ha quedado suficientemente clara: ¿cómo se podría distinguir si lo que parece ser una creencia delirante es realmente una «certeza objetiva individual» o un caso extremo de obstinación, es decir, de certeza subjetiva? A mi entender, la mejor forma de establecer esta diferencia es analizar de forma reiterada múltiples usos del lenguaje del sujeto en cuestión que se refieran indirectamente a la certeza misma. Así pues, el procedimiento adecuado no consistiría en preguntar expresamente al sujeto por su creencia delirante: lejos de tal cosa, habría que comprobar si en diversas situaciones relacionadas con la creencia delirante trata de forma totalmente espontánea cualquier cuestionamiento de dicha certeza no como una duda coherente e inteligible por molesta que pueda resultar, sino como un acto absurdo e incomprensible. Puede haber muchos casos en los que resulte sumamente complejo llevar a cabo esta distinción, así como también puede resultar difícil reformular los conceptos diferenciales sin tener otra referencia que el uso que el sujeto delirante hace del lenguaje: por si fuera poco, a menudo puede ocurrir que este tipo de pacientes apenas hable o que lo que diga resulte prácticamente irrelevante en relación con la tarea que he planteado. Sin embargo, no quiero decir que este procedimiento sea o deba ser útil en todos los casos: simplemente pretendo señalar que en algunas ocasiones puede ayudarnos a conocer mejor el contenido de los delirios. 
Es muy importante señalar que las certezas objetivas individuales no deben ser confundidas con un lenguaje privado, ya que éste sólo se refiere a las sensaciones designadas mediante una definición ostensiva privada. ${ }^{21}$ Por otro lado, hay que admitir que si abundaran las certezas objetivas individuales careceríamos del consenso que permitiría establecer cuándo está gramaticalmente excluida la posibilidad del error; pero es evidente que las certezas objetivas individuales no constituyen sino raras y contadas excepciones que en absoluto afectan a la concordancia general en el uso del lenguaje que dota a éste de significado.

Ya para acabar, considero que la literatura existente sobre este tema se ha visto muy condicionada por la intención de adaptar el fenómeno del delirio a las características que Wittgenstein atribuyó a las certezas, en tanto que yo he procurado hacer justo lo contrario: adaptar e incluso modificar drásticamente algunos rasgos básicos de las certezas entendidas en el sentido wittgensteiniano con el fin de obtener una herramienta conceptual diseñada para ayudarnos a comprender un poco mejor la estructura interna de los delirios. Además, con esta manera de proceder creo haber contribuido también a mostrar el interés de plantearse la existencia de lo que he dado en llamar «certezas objetivas individuales», constructo que puede resultar de utilidad no sólo para conocer con más detalle cómo juegan determinados sujetos nuestros juegos de lenguaje cotidianos, sino también para tener una perspectiva más clara de su sensibilidad y su imagen del mundo.

José María Ariso es Profesor Adjunto en la Universidad Internacional de la Rioja.

Líneas de investigación:

Filosofía de la psiquiatría y teoría del conocimiento.

Publicaciones recientes:

(2011), «Unbegründeter Glaube bei Wittgenstein und Ortega y Gasset: Ähnliche Antworten auf unterschiedliche Probleme», Wittgenstein-Studien, II, pp. 219-248.

(2012), Wahnsinn und Wissen. Zu Wittgensteins Lage und Denkbewegung. Würzburg: Königshausen und Neumann, 2012.

Dirección electrónica: josemaria.ariso@unir.net

$21 C f$. IF $\S 243$. 